Fixed Point Theory, 22(2021), No. 1, 407-430

DOI: $10.24193 /$ fpt-ro.2021.1.28

http://www.math.ubbcluj.ro/ nodeacj/sfptcj.html

\title{
POSITIVE SOLUTIONS FOR FRACTIONAL LAPLACIAN SYSTEM INVOLVING CONCAVE-CONVEX NONLINEARITIES AND SIGN-CHANGING WEIGHT FUNCTIONS
}

\author{
MAODING ZHEN \\ School of Mathematics and Statistics, Huazhong University of Science and Technology, \\ Wuhan 430074, China \\ and \\ School of Mathematics, Hefei University of Technology, Hefei 230009, PR China \\ E-mail: maodingzhen@163.com
}

\begin{abstract}
In this paper, we consider a fractional Laplacian system with both concave-convex nonlinearities and sign-changing weight functions in bounded domains. With the help of the Nehari manifold, we prove that the system has at least two positive solutions when the pair of the parameters $(\lambda, \mu)$ belongs to a certain subset of $\mathbb{R}^{n}$.

Key Words and Phrases: Fractional Laplacian, critical exponent, subcritical exponent, ground state solution, fixed point.
\end{abstract}

2020 Mathematics Subject Classification: 35J50, 35B33, 35R11, 47H10.

Acknowledgements. The author is grateful to Professor Meihua Yang, Jinchun He and Haoyuan Xu for their constructive comments and suggestions, which have greatly improved this paper.

\section{REFERENCES}

[1] A. Ambrosetti, P.H. Rabinowitz, Dual variational methods in critical point theory and applications, J. Functional Anal., 14(1973), 349-381.

[2] G. Alberti, G. Bouchitt, P. Seppecher, Phase transition with the line-tension effect, Arch. Rational Mech. Anal., 144(1998), no. 1, 1-46.

[3] C.O. Alves, D.C. de Morais Filho, M.A.S. Souto, On systems of elliptic equations involving subcritical or critical Sobolev exponents, Nonlinear Anal., 42(2000), no. 5, 771-787.

[4] B. Barrios, E. Colorado, A. de Pablo, U. Sánchez, On some critical problems for the fractional Laplacian operator, J. Differential Equations, 252(2012), no. 11, 6133-6162.

[5] H. Berestycki, P.L. Lions, Nonlinear scalar field equations (I): Existence of a ground state, Arch. Rational Mech. Anal., 82(1983), no. 4, 247-375.

[6] C. Brändle, E. Colorado, A. de Pablo, U. Sánchez, A concave-convex elliptic problem involving the fractional Laplacian, Proc. Roy. Soc. Edinburgh Sect. A, 143(2013), no. 1, 39-71.

[7] H. Brezis, E. Lieb, A relation between pointwise convergence of functions and functionals, Proc. Amer. Math. Soc., 88(1983), no. 3, 486-490. 
[8] X. Cabré, Y. Sire, Nonlinear equations for fractional Laplacians, I: Regularity, maximum principles, and Hamiltonian estimates, Ann. Inst. H. Poincaré Anal. Non Linéaire, 31(2014), no. 1, 23-53.

[9] L. Caffarelli, J. Roquejoffre, Y. Sire, Variational problems with free boundaries for the fractional Laplacian, J. Eur. Math. Soc., 12(2010), no. 5, 1151-1179.

[10] L. Caffarelli, L. Silvestre, An extension problem related to the fractional Laplacian, Comm. Partial Differential Equations, 32 (2007), no. 7-9, 1245-1260.

[11] A. Capella, J. Dávila, L. Dupaigne, Y. Sire, Regularity of radial extremal solutions for some non-local semilinear equations, Comm. Partial Differential Equations, 36(2011), no. 8, 13531384.

[12] X. Chang, Z.-Q. Wang, Ground state of scalar Leld equations involving fractional Laplacian with general nonlinearity, Nonlinearity, 26(2013), no. 2, 479-494.

[13] W.J. Chen, S.B. Deng, The Nehari manifold for a fractional p-Laplacian system involving concave-convex nonlinearities, Nonlinear Anal. Real World Appl., 27(2016), 80-92.

[14] Z. Chen, W. Zou, An optimal constant for the existence of least energy solutions of a coupled Schrödinger system, Calc. Var. Partial Differential Equations, 48(2013), no. 3-4, 695-711.

[15] Z. Chen, W. Zou, Ground states for a system of Schrödinger equations with critical exponent, J. Funct. Anal., 262(2012), no. 7, 3091-3107.

[16] Z. Chen, W. Zou, Positive least energy solutions and phase separation for coupled Schrödinger equations with critical exponent, Arch. Ration. Mech. Anal., 205(2012), no. 2, 515-551.

[17] Z. Chen, W. Zou, Positive least energy solutions and phase separation for coupled Schrödinger equations with critical exponent: higher dimensional case, Calc. Var. Partial Differential Equations, 52(2015), no. 1-2, 423-467.

[18] X. Cheng, S. Ma, Existence of three nontrivial solutions for elliptic systems with critical exponents and weights, Nonlinear Anal., 69(2008), no. 10, 3537-3548.

[19] E. Colorado, A. de Pablo, U. Sánchez, Perturbations of a critical fractional equation, Pacific J. Math., 271(2014), no. 1, 65-85.

[20] A. Cotsiolis, N.K. Tavoularis, Best constants for Sobolev inequalities for higher order fractional derivatives, J. Math. Anal. Appl., 295(2004), no. 1, 225-236.

[21] E. Di Nezza, G. Palatucci, E. Valdinoci, Hitchhiker's guide to the fractional Sobolev spaces, Bull. Sci. Math., 136(2012), no. 5, 521-573.

[22] S. Dipierro, G. Palatucci, E. Valdinoci, Existence and symmetry results for a Schrödinger type problem involving the fractional Laplacian, Le Matematiche, 68(2013), no. 1, 201-216.

[23] R.L. Frank, E. Lenzmann, Uniqueness of non-linear ground states for fractional Laplacians in $\mathbb{R}$, Acta Math., 210(2013), no. 2, 261-318.

[24] R.L. Frank, E. Lenzmann, L. Silvestre, Uniqueness of radial solutions for the fractional Laplacian, Comm. Pure Appl. Math., 69(2016), no. 9, 1671-1726.

[25] Q. Guo, X. He, Least energy solutions for a weakly coupled fractional Schrödinger system, Nonlinear Anal., 132(2016), 141-159.

[26] Z. Guo, S. Luo, W. Zou, On critical systems involving fractional Laplacian, J. Math. Anal. Appl., 446(2017), no. 1, 681-706.

[27] X. He, M. Squassina, W. Zou, The Nehari manifold for fractional systems involving critical nonlinearities, Commun. Pure Appl. Anal., 15(2016), no. 4, 1285-1308.

[28] Y. Hua, M.X. Yu, On the ground state solution for a critical fractional Laplacian equation, Nonlinear Anal., 87(2013), 116-125.

[29] Q. Li, Z. Yang, Multiple positive solutions for a fractional Laplacian system with critical nonlinearities, Bull. Malays. Math. Sci. Soc., 2(2016), no. 4, 1-27.

[30] D.F. Lü, S.J. Peng, On the positive vector solutions for nonlinear fractional Laplacian system with linear coupling, Discrete Contin. Dys. Syst., 37(2017), no. 6, 3327-3352.

[31] J. Marcos, D. Ferraz, Concentration-compactness principle for nonlocal scalar field equations with critical growth, J. Math. Anal. Appl., 449(2017), no. 2, 1189-1228.

[32] A. Mellet, S. Mischler, C. Mouhot, Fractional diffusion limit for collisional kinetic equations, Arch. Ration. Mech. Anal., 199(2011), no. 2, 493-525. 
[33] S.J. Peng, Y.F. Peng, Z.Q. Wang, On elliptic systems with Sobolev critical growth, Calc. Var. Partial Differential Equations, 55(2016), no. 6, art. 142, 30 pp.

[34] S.J. Peng, S. Wei, Q.F. Wang, Multiple positive solutions for linearly coupled nonlinear elliptic systems with critical exponent, J. Differential Equations, 263(2017), no. 1, 709-731.

[35] X. Ros-Oton, Nonlocal elliptic equations in bounded domains: a survey, Publ. Mat., 60(2016), no. $1,3-26$.

[36] R. Servadei, E. Valdinoci, Weak and viscosity solutions of the fractional Laplace equation, Publ. Mat., 58(2014), no. 1, 133-154.

[37] X. Shang, J. Zhang, Y. Yang, Positive solutions of nonhomogeneous fractional Laplacian problem with critical exponent, Commun. Pure Appl. Anal., 13(2014), no. 2, 567-584.

[38] L. Silvestre, Regularity of the obstacle problem for a fractional power of the Laplace operator, Comm. Pure Appl. Math., 60(2007), no. 1, 67-112.

[39] Z. Wang, H.-S. Zhou, Radial sign-changing solution for fractional SchrÖdinger equation, Discrete Contin. Dyn. Syst., 36(2016), no. 1, 449-508.

[40] T.F. Wu, On semilinear elliptic equations involving concave-convex nonlinearities and signchanging weight functions, J. Math. Anal. Appl., 318 (2006), no. 1, 253-270.

[41] T.F. Wu, The Nehari manifold for a semilinear elliptic system involving sign-changing weight functions, Nonlinear Anal., 68(2008), no. 6, 1733-1745.

[42] M.D. Zhen, J.C. He, H.Y. Xu, Critical system involving fractional Laplacian, Commun. Pure Appl. Anal., 1(2019), no. 1, 237-253.

[43] M.D. Zhen, J.C. He, H.Y. Xu, Meihua Yang, Multiple positive solutions for nonlinear coupled fractional Laplacian system with critical exponent, Bound. Value Probl., 2018(2018), Paper no. 96, $25 \mathrm{pp}$.

Received: October 31, 2018; Accepted: July 10, 2019. 